PRINT ISSN 1119-8362

Electronic ISSN 1119-8362
Full-text Available Online at https://www.ajol.info/index.php/jasem

http://ww.bioline.org.br/ja
J. Appl. Sci. Environ. Manage.

Vol. 25 (7) 1155-1161 July 2021

Characterization and Investigation of Nonlinear Behaviour of Radio Refractivity during the Rainy and Dry Seasons in the Coastal Region of Nigeria

\author{
${ }^{1 *}$ ADENIJI, AE; ${ }^{1} \mathrm{AJEIGBE}, \mathrm{EM} ;{ }^{2} \mathrm{OJO}, \mathrm{K} \mathrm{S}$ \\ ${ }^{*}$ Department of Physical Sciences, Bells University of Technology, Ota, Ogun State, Nigeria. \\ ${ }^{2}$ Department of Physics, University of Lagos, Akoka, Lagos State, Nigeria. \\ *Corresponding Author Email: emmaabidec@yahoo.com
}

\begin{abstract}
The transmission and receiving of good quality of signals globally has been impaired due to the internal activities of the troposphere linked to the nonlinear behaviour of the meteorological parameters. This paper focusses on characterizing and investigating the nonlinear behaviour of radio refractivity during the rainy and dry seasons in the coastal region of Nigeria using temperature, pressure and relative humidity data collected over a period of two years by the Centre for Atmospheric Research, National Space Research and Development Agency (NASRDA) from Lagos state. Data obtained were analyzed by False Nearest Neighbour (FNN) and Recurrence Quantification Analysis (RQA). The results show that recurrence techniques are able to identify the periods where there are high predictability and low predictability. The investigation of nonlinear effects of meteorological parameters and the hydrometeors on the troposphere help to avoid fading of radio signals along with the radio communication links.
\end{abstract}

\title{
DOI: https://dx.doi.org/10.4314/jasem.v25i7.8
}

Copyright: Copyright $\odot 2021$ Adeniji et al. This is an open access article distributed under the Creative Commons Attribution License (CCL), which permits unrestricted use, distribution, and reproduction in any medium, provided the original work is properly cited.

Dates: Received: 10 May 2021; Revised: 28 June 2021; Accepted: 01 July 2021

Keywords: Troposphere, recurrence quantification, radio refractivity, predictability

The propagation of electromagnetic waves in the troposphere has been a major challenge due to variation of the atmospheric refractivity. This variation is linked to the turbulence in the troposphere with a high proportion of moisture which causes the tropospheric scatter radio signals to refract and consequently, the receiving antennas collect only a tiny proportion of the radio energy. The atmospheric conditions or different states of the atmosphere at different times, locations and frequencies have significant impact not only degrading the quality of the signal but sometimes completely eradicate the communication link. This impact on Atmospheric refractivity depends solely on atmospheric parameters of air such as pressure, temperature and water content. The weather phenomenon occurs in the troposphere, which is the lowest atmosphere with almost all the atmospheric water vapour content. These atmospheric parameters in most cases appear to be nonlinear considering the variability in their trends. Water vapour concentration (humidity) in the form of hydrometeors, such as fog, clouds or rain, significantly affects microwave frequency above $30 \mathrm{MHz}$, making radio propagation challenging in the tropics, particularly, in the coastline (Zheng and Han-Xian, 2013). The prevalence of sea and land breezes which play a major role in the development and intensification of weather events in the coastal cities accounts for the high concentration of water vapour (Gadgil, 2003; Leroyer et al., 2014; Sikka and Gadgil, 2003, 1980). It is generally agreed that the radiometeorological situation in the coastal areas can produce complicated structures due to the interaction between the sea and the land (Castel, 1965). Hence, it is useful to further investigate into this field since there is still insufficient information about the nonlinear behavior of these atmospheric parameters. The effect of atmospheric refractivity on the propagation of radio waves has been studied from the beginning of radio wave technology (Kerr, 1987). It has been established that the refraction of electromagnetic waves due to inhomogeneous spatial distribution of the refractive index of air causes adverse effects such as multipath fading and interference, attenuation due to diffraction on the terrain obstacles or so-called radio holes (Lavergnat and Sylvain, 2000; Adediji and Ajewole, 2008). The anomalous propagation is due to the variations of the humidity, temperature and pressure at the atmosphere that cause variations in the refractive index (Norland, 2006). The climatic conditions are very changeable and unstable in Lithuania (Pankauskas and Bukantis, 2006). Some works have been carried out on radio refractivity using nonlinear dynamics and chaotic approaches. Ogunjo et al. (2013) showed the existence of chaos in radio refractivity at ground level. Adediji and Ogunjo 
(2014) reported chaotic behaviour of radio refractivity values at different heights (Ground surface, $50 \mathrm{~m}, 100$ $\mathrm{m}$ and $150 \mathrm{~m}$ ) over a period of five years in the tropical station, Akure, South-Western Nigeria. Ogunjo et al. (2016) investigated chaos in surface radio refractivity in eleven locations over Nigeria using Brock- DechertScheinkmen (BDS) test and reported that the BDS values in the dry season for surface radio refractivity are in a state of chaos while surface radio refractivity in rainy seasons was found to be regular. The degree of the complexity and nonlinear trends of Radio Refractivity Gradient (RRG) in the troposphere over selected locations in Nigeria were investigated (Adelakun et al., 2019; Ojo et al., 2019). Fuwape and Ogunjo (2016), also considered different quantification parameters including the Hurst exponents, LLE correlation dimension, recurrence quantification analysis (RQA) for the analysis of the forecast data from (ECMWF) and (ERA- interim) project. They were able to validate the efficacy of the RQA and also characterized the data based on other parameters.

In the study of refractive index turbulence, it was observed that within the atmospheric boundary layer the refractive index fluctuates chaotically in time (Wyngaard and Le Mone, 1980; Fairall, 1987; Wyngaard et al., 2001). This chaotic fluctuation is due to the variations of the humidity, temperature and pressure in the atmosphere. These meteorological parameters are made up of wet and dry components of the radio refractivity which cause climatic conditions to be very changeable and unstable. Therefore, there is need to investigate the chaotic behaviour of the wet and dry components of the refractive index as the effect of this behaviour can impair radio propagation which cannot be ignored by the designers of communication systems. This paper focusses on characterizing and investigating the nonlinear behaviour of radio refractivity during the rainy and dry season in the coastal region of Nigeria.

\section{MATERIALS AND METHODS}

The study area is located in Lagos which is one of the coastal regions in Nigeria. It lies between the Latitude $6.434389^{\circ} \mathrm{N}$ and longitude $3.322639^{\circ} \mathrm{E}$. The climatic is characteristics into wet and dry seasons in which the wet season starts from March and end in October every year while dry season is between October and March every year. The temperature, pressure and relative humidity data used were collected by the Centre for Atmospheric Research, National Space Research and Development Agency (NASRDA) from Lagos state and recorded cover 24 hours each day from 00:00 hours to 23:00 hours local time at 5 minutes interval for the period of two years from January 1, 2011 -
December 31, 2012. The wet and dry components of radio refractivity was categorized into rainy and dry seasons which were used for the analysis.

Radio Refractivity: The refractive index, $n$, can be expressed in terms of radio refractivity, $\mathrm{N}$ as follows (Smith and Kroll, 2000):

$n=1+N \times 10^{6}$

The multiplication factor in equation (1) was used because the refractive index, $n$, for air deviates from unity by at most a few parts per ten thousand. Radio refractivity, $(\mathrm{N})$, which is the sum of the dry and wet parametric values, can be expressed as

$N=77.6 \frac{P}{T^{2}}+3.73 \times 10^{5} \frac{e}{T^{2}}$

The Clausius-Clapeyron relationship between water vapor pressure, e $(\mathrm{hPa})$, saturation vapor pressure, $e_{s}$ $(\mathrm{hPa})$ and relative humidity, $\mathrm{H}(\%)$, can, therefore, be deduced from equation (2) as

$e=\frac{H}{100} e_{S}$

where $e_{s}=a \exp \frac{b t}{t+c}$

The parameters in (4) are derived as $a=6.1121, b=$ 17.502, $c=240.97$. $\mathrm{T}$ is the atmospheric temperature in $\mathrm{K}$, dry atmospheric pressure $\mathrm{P}$ in $\mathrm{hPa}$, and $\mathrm{t}$ is the temperature in ${ }^{0} \mathrm{C}$.

Determination of embedding dimension and time delay: In the phase space reconstruction and recurrence analysis from a time series, two essential parameters are necessary to be estimated. These parameters are delay time $(\tau)$ and embedding dimensions (m) which can be widely found in the literature (e.g., Kantz and Schreiber, 1997). The optimal time delay $\tau$ can be estimated as the first minimum of the mutual information (MI) function using equation (5). In nonlinear time series analysis, the mutual information (MI) function is defined in Takens (1981).

$I(r)=-\sum_{i j} P_{i j} \operatorname{In} \frac{P_{i j(r)}}{P_{i} P_{j}}$

Where $P_{i}$ is the probability of finding a time series value in the $i^{\text {th }}$ interval in the partition and $P_{i j}(\tau)$ is the joint probability to find a time series value in the $i^{\text {th }}$ interval and a time series value in the $j^{\text {th }}$ interval after a time $\tau$. Kennel et al., (1992) proposed a common method to determine the minimal sufficient embedding dimension $\mathrm{m}$ which is the false nearest 
neighbour (FNN) method. The percentage of false nearest neighbours (FNN) is estimated for each embedding dimension $\mathrm{m}$ of a set of time series data and the first percentage of false nearest neighbours (FNN) where it drops to zero is chosen as the embedding dimension.

Recurrence quantification analysis: The hidden patterns contained in the recurrence plot cannot be easily ascertained by visual inspection. Zbilut and Webber (1992) proposed a tool called recurrence quantification analysis (RQA) to solve the problem of hidden patterns in RPs by quantifying the presence of patterns in the RPs. The measures to quantify the deterministic structure and chaoticity of RPs used in this research work include the following:

(i) Determinism (DET) is the ratio of recurrence points forming diagonal structures (of at least length $l_{\text {min }}$ ) to all recurrence points.

$D E T=\frac{\sum_{l=l_{\min }} l P(l)}{\sum_{i j}^{N} R_{i j}}$

Where $(l)$ is the frequency distribution of the diagonal line lengths (for a diagonal parallel to the main diagonal); $l$ is the length of the line structure.

(ii) Divergence (DIV) is defined as the inverse of the length Maxline $\left(L_{\max }\right)$ of the longest diagonal line segment measured parallel to the main diagonal in the RP. It indicates how rapidly parallel trajectories diverge from one another. The shorter the longest line is, the more divergent the trajectories will be. This was proposed by Eckmann et al., (1987) that the longest diagonal line structure was inversely proportional to the most positive Lyapunov exponent. A periodic signal will give long line segments, while short lines indicate chaos.

$$
D I V=\frac{1}{L_{\max }}, L_{\max }=\max \left(\left\{l_{i} ; i=1, \ldots N_{l}\right\}\right)
$$

$N_{l}$ is the total number of diagonal lines.

\section{RESULTS AND DISCUSSION}

The detailed analysis of Embedding time delay and Embedding Dimension of the wet and dry components of the radio refractivity for the period of dry and wet seasons from January, 2008 to December, 2009 are given using average mutual information and percentage of the false nearest neighbour. The values of the Embedding time delay and Embedding Dimension are used in the analysis of Phase Space Reconstruction and Recurrence Quantification Analysis (RQA). Figures 1 and 2 show the average mutual information against the embedding time delay and the percentage of false nearest neighbour against the embedding dimension with the first minimum of the embedding time delay $(\tau)$ observed at 6 and optimum embedding dimension $m$ at 12 respectively. It is observed for the 24 months of the two years that a choice of time delay of 6 and embedding dimension of 12 are the suitable choices for $\tau$ and m, respectively by following the concept in Wallot (2017) and Adeniji et al., (2018), in which a single value that seems to have good characterization across both years is picked.

In order to unfold the attractors and to avoid underembedding one system than the other, we choose the embedding dimension from a system with higher dimension. Thus, the choice of $\tau$ as 6 and $m$ as 12 for further analysis of this observation are reasonable.

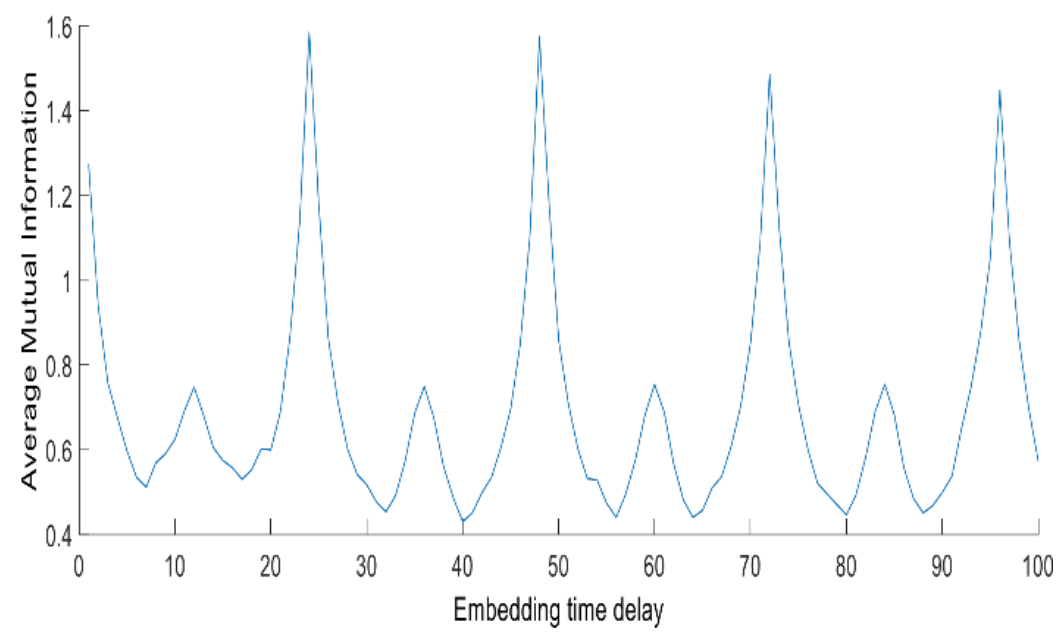

Fig 1: Average Mutual information versus Time delay $(\tau)$ 


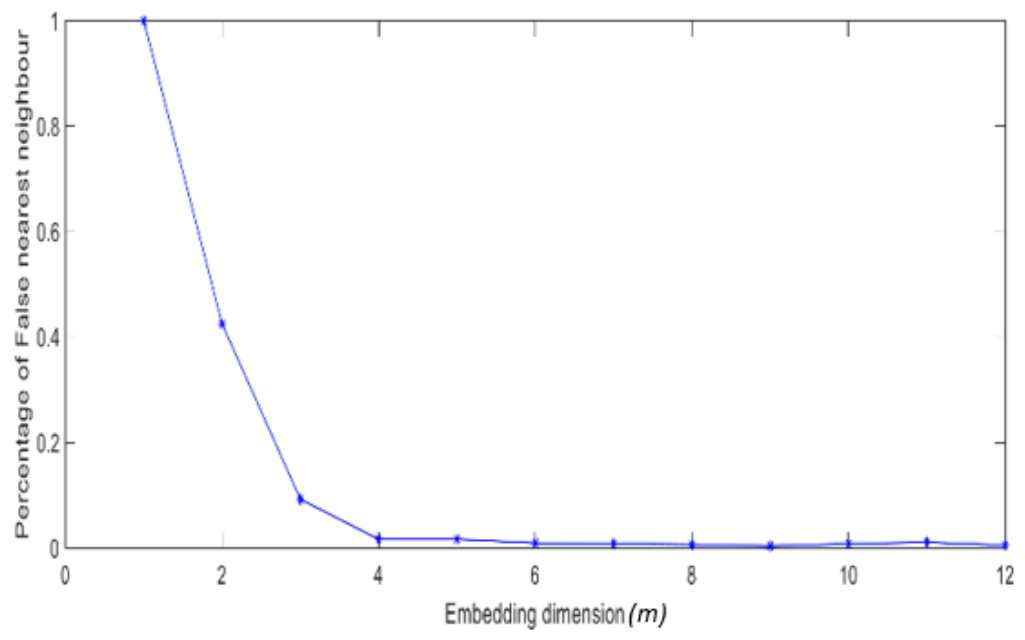

Fig 2: Percentage of False Nearest Neighbour versus Embedding Dimension $(m)$.

The quantitative analysis technique of the recurrence quantification analysis (RQA) method gives detailed information of the nonlinear dynamical system. There are two RQA measures used in this research work, these are Divergence (DIV) and Determinism (DET). The results of the two quantifiers DIV and DET are shown in figures 3 and 4 , respectively.

The divergence (DIV) values of the wet component of radio refractivity for year 2008 ranges from 0.003802 to 0.200000 and that of year 2009 ranges from 0.002809 to 0.200000 while the dry component of the radio refractivity ranges from 0.00289 to 0.066667 for year 2008, and that of year 2009 ranges from 0.002841 to 0.013699 . We also observed that the DIV values decrease at the beginning of the year and the values increase gradually towards the last quarter of the year (August) where most regions experience maximum rainfall and thereafter begin to decrease again at the return of the dry season. In figure 3 , the variation of the level of chaoticity of the wet and dry components in both the dry and wet seasons is shown. We observed that the wet component of May 2008 and August 2009 which are wet season months, exhibited high chaoticity while the remaining months exhibited lower chaoticity when compared. The dry season months of the wet and dry component of both years exhibited low chaoticity. The high chaoticity exhibited may be as a result of high influence of meteorological parameters such as relative humidity, temperature, pressure, and water vapour density during the wet season of both years which also increases the complexity of the troposphere. The major meteorological parameter that could cause the high chaoticity is attributed to high concentration of water vapor (Adeniji et al., 2020; Osinowo and Okogbue, 2014). The low chaoticity exhibited may be as a result of low influence of the meteorological parameters on the wet and dry components of the radio refractivity during dry season. The effect of low concentration of water vapour on the wet and dry components of the radio refractivity for the two years could be the major cause of the low chaoticity. Refractivity is fairly stable and high during the rainy season (April to September) (Agbo et al., 2020) which implies high chaoticity. The figure 3 also shows that the dry season (January to March, October to December) has low chaoticity which agrees with Adeniji et al., 2018 and has a low refractivity (Agbo et $a l ., 2020)$. Figure 4 shows the DET values of the wet component of radio refractivity for year 2008 ranges from 0.0884243 to 0.089987 and that of year 2009 ranges from 0.435328 to 0.875404 . It is observed that the dry component of the radio refractivity ranges from 0.580052 to 0.868199 for year 2008 , while that of year 2009 ranges from 0.48992 to 0.845623 . High determinism (predictability) values could be found in the dry season months of both years, the highest value is observed in the month of January 2009 and the lowest is observed in the month of June, 2008. Figure 4 depicts the results of determinism, the results exhibit the same trend with high values during the dry months and low values in wet months. High determinism (high predictability) values could be found in the wet and dry components of the radio refractivity of both years in the dry season. The highest determinism (high predictability) value is found in the wet component of the radio refractivity of the dry season in January 2009 while the lowest determinism (low predictability) value is found in the wet component of the radio refractivity of the wet season in the month of August 2009. High length of predictability of the wet and dry components of the radio refractivity during the dry season will enhance the transmission and receiving of good quality of signals. 


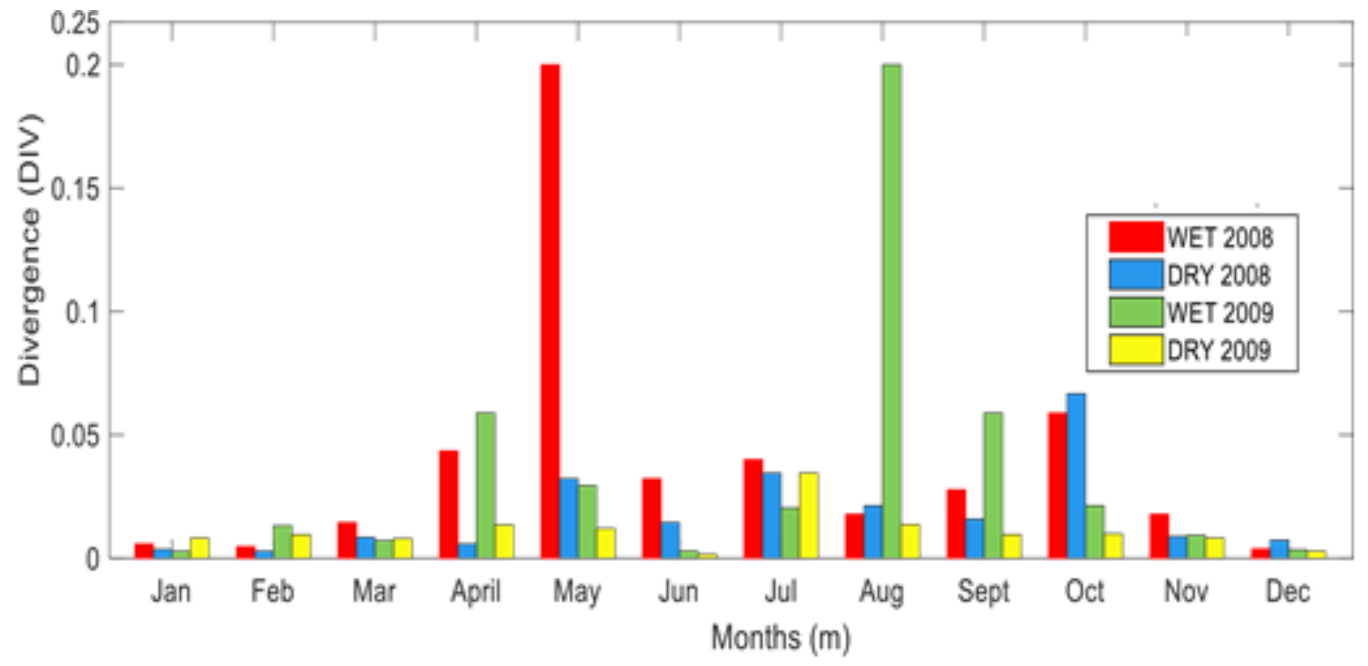

Fig 3: Divergence of the wet and dry components of radio refractivity for both years

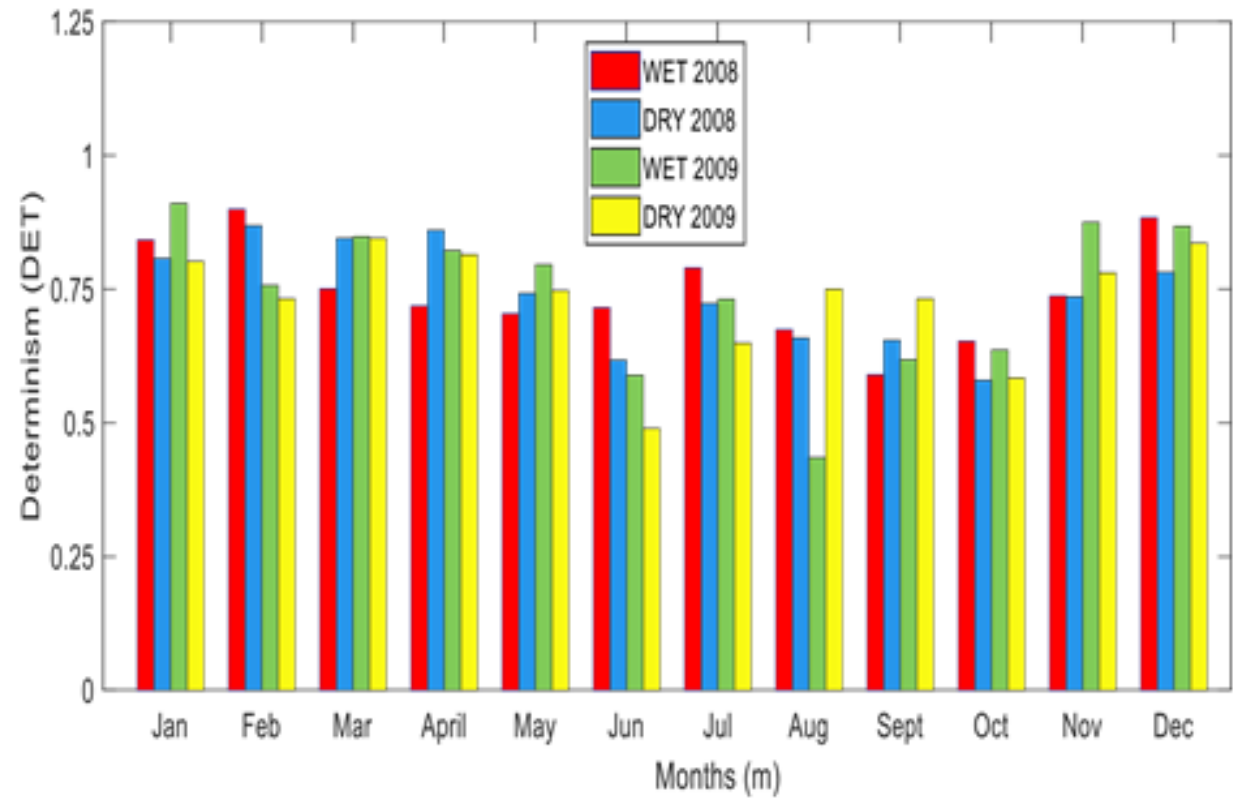

Fig 4: Determinism of the wet and dry components of radio refractivity for both years

Conclusion: In this study, the wet and dry components of the radio refractivity, the rainy and dry seasons for two years (2008 and 2009) were studied separately using the methods False nearest neighbour (FNN) and recurrence quantification analysis (RQA). The dimensionalities of the wet and dry components of the radio refractivity were evaluated and the underlying dynamics was quantified. The nonlinear behavior of the wet and dry components of the radio refractivity were observed which will help to avoid fading of radio signals along with the radio communication links.

\section{REFERENCES}

Adediji, AT; and Ajewole, MO (2008). Vertical profile of radio refractivity gradient in Akure south-west Nigeria. Progress in Electromagnetics Research C 4:157-168.

Adediji, AT; Ogunjo, ST (2014). Variations in NonLinearity in Vertical Distribution of Microwave Radio Refractivity. Progress in Electromagnetics Research M 36: 177-183.

Adelakun, AO; Ojo, JS; Edward, OV (2019). Quantitative Analyses of Complexity and Nonlinear trend of Radio Refractivity Gradient in the troposphere. Adv. Space Res. doi: https://doi.org/10.1016/j.asr.2019.09.055. 
Adeniji, AE; Njah, AN; Olusola, OI (2020). Regional and Seasonal Variation of Chaotic Features in Hourly Solar Radiation Based on Recurrence Quantification Analysis. J. Appl. Nonlinear Dyn. 9(2):175-187.

Adeniji, AE; Olusola, OI; Njah, AN (2018). Comparative study of chaotic features in hourly wind speed using recurrence quantification analysis. AIP Advs. 8: 2158- 3226.

Agbo, E P; Ettah, E B; Eno, E E (2020). The impacts of meteorological parameters on the seasonal, monthly, and annual variation of radio refractivity. Indian J. Phys. PACS Nos.: 07.05. Kf; 07.05.Hd; 06.20.-f. https://doi.org/10.1007/s12648-02001711-9

Castel, FD (editor) (1965): "Progress in Radio Science", Elsevier Publishing Company, 19601963 Volume 2 - Radio and Troposphere, pg. $127-$ 135.

Eckmann, J P; Kamphorst, S; Ruelle, D (1987). Recurrence plots of dynamical systems. Europhys. Lett. 4: 973-977.

Fairall, CW (1987). A top down and bottom-up diffusion model of $\mathrm{C}_{\mathrm{T}}{ }^{2}$ and $\mathrm{C}_{\mathrm{Q}}{ }^{2}$ in the entraining convective boundary layer. J. Atmos. Sci. 44: 10091017.

Fuwape, IA; Ogunjo, ST (2016). Quantification of scaling exponents and dynamical complexity of microwave refractivity in a tropical climate. Jour. of Atmos. and Solar-Terrestrial Physics 150: 6168.

Gadgil, S (2003). The Indian Monsoon and its Variability. Annu. Rev. Earth Planet. Sci. 3: 429467.

Kantz, H; Schreiber, T (1997). Nonlinear Time Series Analysis, University Press, Cambridge.

Kennel, MB; Brown, R; Abarbanel, HDI (1992). Determining embedding dimension for phase space reconstruction using a geometrical reconstruction. Phys Rev A. 45(2): 3403- 3411.

Kerr, DE (Ed.) (1987). Propagation of Short Radio Waves, Peter Peregrinus Ltd./IEE, ISBN 0- 86341099-5, London, UK.

Lavergnat, J; Sylvain, M (2000). Radio Wave Propagation: Principles and Techniques, John
Wiley \& Sons., ISBN 0-471-49027-X, Chichester, UK.

Leroyer, S; B’eLair, S; Husain, SZ; Mailhot, J (2014). Subkilometer Numerical Weather Prediction in an Urban Coastal Area: A Case Study over the Vancouver Metropolitan Area. J. Appl. Meteor. Climatol. 56 (6): 1433-1453.

Norland, N (2006). Temporal Variation of the Refractive Index in Coastal waters. Proceedings of IRS 2006, International Radar Symposium, pp. 221-224, Krakow, Poland, 24 - 26 May 2006, ISBN 83-7207-621-9.

Ogunjo, ST; Ojo, J S; Adediji, AT; Adedayo, KA; Dada, JB (2013). Chaos in radio refractivity over Akure, South- Western Nigeria. In 5th Annual Conference of the Nigeria Union of Radio Science (NURS) pp 56-63.

Ogunjo, ST; Fuwape, IA; Oluyamo, SS; Akinpelu, SB (2016). Complexity in Seasonal Dynamics of surface radio refractivity across different climate zones of Nigeria. URSI Asia- Pacific Radio Science Conference, August 21- 25, 2016/ Seoul, Korea.

Ojo, JS; Adelakun, AO; Edward, OV (2019). Comparative study on Radio Refractivity Gradient in the troposphere using Chaotic Quantifiers. Heliyon. 5, e02083.

Osinowo, AA; Okogbue, CE (2014). Investigation of the sky optical conditions over selected stations in the major vegetation zones of Nigeria. Int. J. Sustain. Energy. 33(2): 357-366.

Pankauskas, M; Bukantis, A (2006). The dynamics of the Baltic Sea Region climate humidity. Annales Geographicae. 39(1): 5-14.

Smith, DR; Kroll, N (2000). Negative Refractive Index in Left-Handed Materials. Phys. Rev. Lett. 85: 2933-2936.

Sikka, D; Gadgil, S (2003). On the maximum cloud zone and the ITCZ over Indian longitudes during the southwest monsoon. Mon. Wea. Rev. 108: 1840-1853.

Sikka, D; Gadgil, S (1980). Large-scale rainfall over India during the summer monsoon and its relation to the lower and upper tropospheric vorticity. Indian J. Meteor., Hydro. and Geophys. 29: 219231.

Takens, F (1981). Dynamical systems and turbulence, lecture notes in mathematics, Springer, London, 
898, pp 366-381. Detecting strange attractors in turbulence.

Wyngaard, JC; Nelson, SS; Otte, M; Xaio D; Kenneth, EG (2001). Concepts, observations, and simulation of refractive index turbulence in the lower atmosphere. Radio Science. 36: 643-669.

Wyngaard, JC; LeMone, MA; (1980). Behaviour of the refractive index structure parameter in the entraining convective boundary layer. J. Atmos. Sci. 37:1573- 1585 .
Wallot, S (2017). Recurrence Quantification Analysis of processes and products of discourse: A tutorial in R. Discourse Processes (Taylor and Francis Group): 54: 382- 405.

Zbilut, J; Webber, JC (1992). Embeddings and delays as derived from quantification of recurrence plots. Phys. Lett. A 171:199 - 203.

Zheng, S; Han-Xian, F (2013). Monitoring of Ducting by using a ground based GPS receiver. Chin. Phys. B. 22 (2):1-5. 